\title{
Prevalence of Vancomycin resistant enterococci (VRE) in Ethiopia: a systematic review and meta-analysis
}

\author{
Addisu Melese $^{1 *}$ (D) Chalachew Genet ${ }^{1}$ and Tesfaye Andualem²
}

\begin{abstract}
Background: The emergence of Vancomycin resistant enterococci (VRE) poses a major public health problem since it was first reported. Although the rising rates of VRE infections are being reported elsewhere in the worldwide; there is limited national pooled data in Ethiopia. Therefore, this study was aimed to estimate the pooled prevalence of VRE and antimicrobial resistance profiles of enterococci in Ethiopia.

Methods: Literature search was done at PubMed, EMBASE, Google scholar, African Journals online (AJOL) and Addis Ababa University repository following the Preferred Reporting Items for Systematic Reviews and Meta-Analyses (PRISMA) guideline. Both published and unpublished studies reporting the prevalence of VRE until June 30, 2019 were included. Data were extracted using Microsoft Excel and copied to Comprehensive Meta-analysis (CMA 2.0) for analysis. Pooled estimate of VRE was computed using the random effects model and the $95 \% \mathrm{Cls}$. The level of heterogeneity was assessed using Cochran's $Q$ and $I^{2}$ tests. Publication bias was checked by visual inspection of funnel plots and Begg's and/or Egger's test.
\end{abstract}

Results: Twenty studies fulfilled the eligibility criteria and found with relevant data. A total of 831 enterococci and 71 VRE isolates were included in the analysis. The pooled prevalence of VRE was $14.8 \%\left(95 \% \mathrm{Cl} ; 8.7-24.3 ; P^{2}=74.05 \% ; P<0.001\right)$. Compared to vancomycin resistance, enterococci had higher rate of resistance to Penicillin (60.7\%), Amoxicillin (56.5\%), Doxycycline (55.1\%) and Tetracycline (53.7\%). Relatively low rate of resistance was found for Daptomycin and Linezolid with a pooled estimate of 3.2\% (95\% Cl, 0.5-19.7\%) and 9.9\% (95\% Cl, 2.8-29.0\%); respectively. The overall pooled multidrug resistance (MDR) rate of enterococci was $60.0 \%$ (95\% Cl, 42.9-75.0\%).

Conclusion: The prevalence of VRE and drug resistant enterococci are on the rise in Ethiopia. Enterococcal isolates showed resistance to one or more of the commonly prescribed drugs in different or the same drug lines. Multidrug resistant (MDR) enterococci were also found. Although the rates were low, the emergence of resistance to Daptomycin and Linezolid is an alarm for searching new ways for the treatment and control of VRE infections. Adherence to antimicrobial stewardship, comprehensive testing and ongoing monitoring of VRE infections in the health care settings are required.

Keywords: Enterococcus, Vancomycin resistance, Systematic review, Ethiopia

\footnotetext{
* Correspondence: addisum22@gmail.com

'Department of Medical Laboratory Science, College of Medicine and Health

Sciences, Bahir Dar University, Bahir Dar, Ethiopia

Full list of author information is available at the end of the article
}

(c) The Author(s). 2020 Open Access This article is distributed under the terms of the Creative Commons Attribution 4.0 International License (http://creativecommons.org/licenses/by/4.0/), which permits unrestricted use, distribution, and reproduction in any medium, provided you give appropriate credit to the original author(s) and the source, provide a link to the Creative Commons license, and indicate if changes were made. The Creative Commons Public Domain Dedication waiver (http://creativecommons.org/publicdomain/zero/1.0/) applies to the data made available in this article, unless otherwise stated. 


\section{Background}

Today, antimicrobial resistance (AMR) is one of the most important public health problem in the world and continues to challenge treatment especially in bacteria [1]. Widespread use and misuse of antibiotics is thought to increase the prevalence and emergence of resistance bacterial strains. As a growing problem; AMR complicates the treatment of bacterial infections leading to increased mortality, morbidity and healthcare related costs. The emergence of Vancomycin resistant enterococci (VRE) poses a major public health problem since it was first reported. VRE are among the most common resistant pathogens frequently causing healthcare associated infections and a growing concern for health care professionals [1-4].

Enterococci are gram-positive bacterial flora of the intestinal tract of humans, animals and birds [5-7]. Despite their commensal characteristics, they cause serious nosocomial infections in humans including urinary tract, bloodstream infections and endocarditis [8]. They are "tough bugs" that can survive in/and on the environment for long periods and became one of the main nosocomial pathogens. Enterococci are also able to form biofilms that contribute to the virulence, resistance to antibiotics and phagocytosis making their eradication extremely difficult $[9,10]$.

Enterococci become resistant to a variety of antimicrobials through intrinsic and acquired mechanisms. Isolates of E. gallinarum and E. flavescens develop an inherent, low-level resistance to Vancomycin [11]. Enterococci readily accumulate mutations and exogenous genes that confer additional resistance. They develop resistance to vancomycin by exchange of genetic material among themselves and/or with another genera [12]. The enterococci may acquire resistance through van associated genetic elements (vanA, vanB, vanD, vanE, vanG, vanL); of which vanA and $v a n B$ are the most prevalent genotypes in clinical isolates $[11,13,14]$. The $v a n A$ and $\operatorname{van} B$ gene clusters are most commonly found in E. faecium and increasingly reported throughout the world $[12,15]$. Other transposable elements are also reported to be involved in the spread of antimicrobial resistance [16].

Vancomycin was considered as one of the last lines of treatment against multidrug resistant organisms including ampicillin resistant enterococci and methicillin-resistant Staphylococcus aureus (MRSA) [8]. However, enterococci develop high level of resistance and the incidences of VRE infections among hospitalized patients has increased rapidly [9, 13, 17]. Infections due to VRE have been also reported to be associated with longer hospital stays, increased mortality and higher healthcare costs than infections with vancomycin susceptible enterococci $[15,18-20]$.

Enterococcal infections are now getting attention due to their ability to develop resistance to multiple antimicrobial agents which probably explain their large part of isolation in nosocomial infections [21, 22]. The two species (E. faecalis and E. faecium) are responsible for majority of the infections in humans. They are also constituting a reservoir for antibiotic resistance among the gut enterococci [23]. In 2017, the World Health Organization (WHO) has published the priority lists of antibiotic-resistant bacteria to guide research, discovery, and development of new antibiotics. Hence; Vancomycin resistant E. faecium was categorized as high priority pathogens for which new and effective treatments are need [24]. Reports are also emerging on the development of resistance to Daptomycin and Linezolid which are being used to treat Vancomycin resistant enterococcal infections [14]; this could explain the challenging nature of these bacteria in the current medicine and as well as to the future. Other studies reported the continuous increase of VRE causing nosocomial infections [25].

In Ethiopia; different reports showed that antimicrobials are widely misused by health care providers, unskilled practitioners, animal husbandry operators and drug users. Antimicrobial misuse is one of the major driver and contributor of the emergence and survival of resistance strains. To prevent and contain the spread of drug resistance, the Ethiopian Public Health Institute (EPHI) established AMR surveillance centers and identified national priority surveillance pathogens in 2017 [4]. A previous systematic review has also reported the growing challenges of antibacterial drug resistance in Ethiopia [26]; but VRE were included neither in the national priority surveillance pathogens nor in previous systematic reviews. Although the rising rates of VRE infections are being reported elsewhere in the worldwide; there is limited national pooled data in Ethiopia. Therefore; this study was aimed at summarizing the findings of local studies to estimate the pooled prevalence of VRE and antimicrobial resistance profiles of enterococci in Ethiopia.

\section{Methods \\ Search strategy}

A comprehensive search was conducted at PubMed, EMBASE, Google scholar and African journals online (AJOL). To include unpublished studies (theses, dissertations); the repository of Addis Ababa University was searched. Reference lists of included studies were also sought. The database search was done following the PRISMA guideline/checklists [27] (Fig. 1). The PubMed was searched using MeSH terms and Boolean operators. The search string in PubMed was: $\left(\left(()\left(\left(\left(\right.\right.\right.\right.\right.$ Enteroccoc $\left.\left.^{*}\right)\right)$ OR Enterococcus faecalis) OR Enterococcus faecium) OR E. faecalis OR E. faecium AND Vancomycin resistan*) OR antibiotic resistan*) OR antimicrobial resistan*) OR drug resistan*) OR VRE) AND Ethiopia)))))))). Search results were combined in to EndNote X6 (Clarivate Analytics USA) and duplicates were removed. Studies published/ 


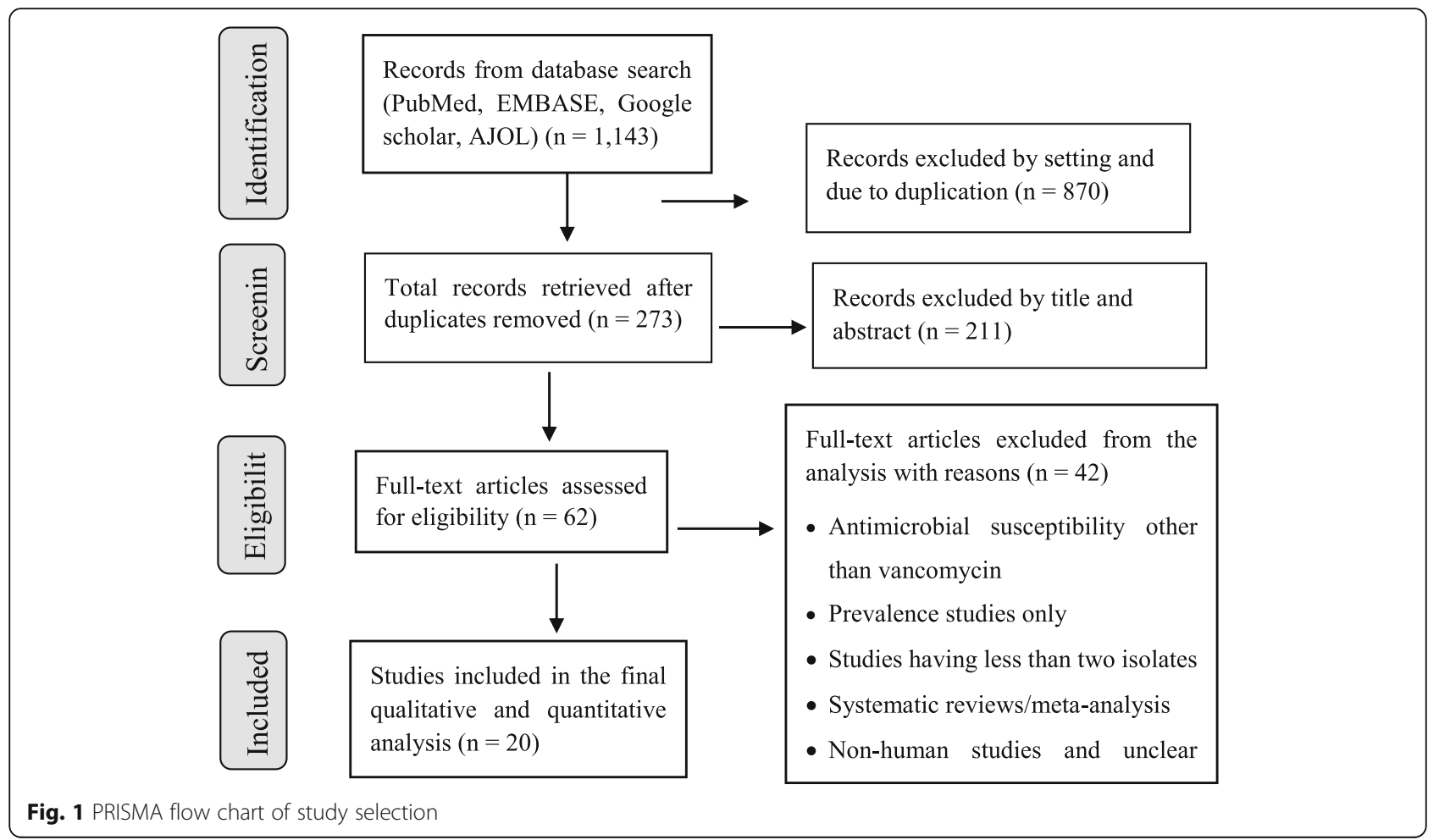

reported up to June 30, 2019 and fulfilled the eligibility criteria (Table 1) were included.

\section{Quality assessment}

The quality of included studies was assessed by the Joanna Briggs Institute (JBI) critical appraisal checklist for prevalence data [28] (additional file 1); which contains nine sections. The assessment was done independently by two authors (AM and TA). Studies were included in the analysis if consensus was reached among the two reviewers. The quality of the 20 included studies is given in (additional file 2).

\section{Data extraction}

After studies were identified based on the predefined eligibility criteria; author name with year of publication, study period, region of study, study design, sample size, study population, types of specimens, antimicrobial susceptibility testing (AST) methods, number of isolates (both the total and vancomycin resistant enterococci), types of isolated species and history of publication were extracted using Microsoft Excel 2013 data collection sheet especially designed for this study. Resistance profiles of enterococci to other antimicrobials were also extracted and the study level proportions were pooled. The data extraction was done independently by two authors (AM and TA).

\section{Data analysis}

Whenever studies were not reporting the prevalence of VRE, it was calculated by dividing the numbers of VRE isolates to the total numbers of tested enterococcal isolates and multiplying by 100 . Studies reporting a zero number of VRE isolates were imputed to 0.5 as a continuity correction to be include in the meta-analysis [29]. Subgroup analyses were done by the study region, study period, publication history, AST and types of specimens used to isolate enterococci.

Table 1 Eligibility criteria

\begin{tabular}{ll}
\hline Inclusion criteria & Exclusion criteria \\
\hline - Study settings: conducted in Ethiopian on any settings & - Studies on antimicrobial susceptibility tests other than vancomycin \\
- Study subjects/population: humans & (studies that did not include VRE) \\
- Study design: any study reported the prevalence of VRE or & - Prevalence studies only \\
numbers of VRE and total enterococci isolates & - Studies having less than two isolates \\
- Sample size: studies isolated not less than two enterococci & - Studies not reporting enterococcal isolates separately \\
- Language: published/reported in English & (no population denominator) \\
- Type of study: peer-reviewed, full text available before & - Reviews, comments and duplications \\
June 30,2019 & - Studies on non-human subjects \\
\hline
\end{tabular}


Acknowledging the presence of heterogeneity in observational studies conducted in diverse settings, the random effects model was used in determining the pooled prevalence of VRE as well as resistance to other antimicrobials. Heterogeneity was evaluated by the Cochran's Q-test and $\mathrm{I}^{2}$ statistics. Funnel plots were drawn to see the presence of publication bias and the Begg's rank correlation and Egger's regression tests were used to quantify the degree of publication bias. P-values $<0.05$ in any of the Begg's rank correlation and Egger's regression tests were indicative of significant publication bias. In asymmetrical funnel plots, the Trim-and-Fill method was applied to include missing studies and estimate adjusted effect sizes. Sensitivity analysis in a leave-one-out approach was done to see the stability of the pooled prevalence of VRE and to explore the potential source of heterogeneity between studies. Data were analyzed using CMA version 2.0 for windows and used to generate forest and funnel plots.

\section{Results}

Study selection

The results of database search and process of study selection is shown in the flow chart below (Fig. 1). The search returned 1143 records; of which 62 studies were subjected for full text review for inclusion against the eligibility criteria. Finally, 42 studies were excluded and only 20 were included in our analysis.

\section{Characteristics of included studies}

All of the 20 studies included in this review were crosssectional by design. Most of the studies were reported from Amhara region $(n=8)$ [30-37] and Addis Ababa $(n=7)$ [38-44]. The remaining studies were from Oromia $(n=4)$ [45-48] and Southern nations $(n=1)$ [23]. Studies were not available from administrative regions of Tigray, Afar, Dire Dawa, Harari, Somali, Gambela and BenishangulGumuz. Nineteen studies were conducted in hospital settings. Among the 6017 study participants included, 831 enterococci were isolated and tested with a variety of antimicrobials; of which 71 isolates were resistant to vancomycin. Stool, urine, blood and swab specimens were used to isolate enterococci. The highest numbers of enterococcal and VRE isolates were identified from stool followed by multi-site specimens.

Seventeen studies used disc diffusion and three studies employed dilution/minimum inhibitory concentration (MIC) as antimicrobial susceptibility testing (AST) method to determine Vancomycin resistance. Resistance to antimicrobial agents by either methods was defined based on the performance standards for antimicrobial susceptibility testing guidelines prepared by Clinical and Laboratory Standards Institute (CLSI, various editions). The prevalence of VRE ranged from $1.8 \%$ in Jimma to $60 \%$ in Addis Ababa. Species level enterococci were reported by four studies [23,
39, 47, 48] and E. faecalis and E. faecium were the most frequently isolated species. Six of the included studies were unpublished and 14 were published between 2013 and 2019. Details of the characteristics of the included studies is summarized in (Table 2) below.

\section{Pooled prevalence of VRE}

The pooled prevalence of VRE was estimated at $14.8 \%$ (95\% CI; 8.7-24.3\%; $I^{2}=74.05 \% ; P<0.001$ ) (Fig. 2). Significant heterogeneity $\left(Q=73.21 ; I^{2}=74.05 \% ; P<0.001\right)$ was observed in the estimation of overall pooled result. But, the sensitivity analysis revealed that no single study significantly influenced the heterogeneity and pooled prevalence of VRE. The pooled prevalence of VRE in the sensitivity analysis ranged from 13.2 to $16.7 \%$ which lies within the $95 \%$ CI bounds of the overall pooled estimate. The presence of publication bias was observed from the drawn asymmetric funnel plot (Fig. 3a). The Trim-and-Fill method was then applied to include the "missing" studies from the analysis. The asymmetric studies were trimmed to locate the unbiased effect and fills the plot by re-inserting the trimmed studies as well as their imputed counterparts. Accordingly, one study was missed and fall at the left side of the pooled estimate (Fig. 3b). In the Trim-and-Fill method, the adjusted estimate of VRE was 13.5\% (95\% CI; 7.8-22.2\%); almost similar with the original pooled estimate. The Egger's regression (intercept $=0.91 ; 95 \% \mathrm{CI}$; $0.75-2.57 ; p=0.263)$ and Begg's rank test $(p=0.381)$ did not suggest significant publication bias.

\section{Subgroup prevalence of VRE}

The prevalence of VRE was computed by region, type of antimicrobial testing (AST) method, study period, types of specimen used to isolate enterococci, and publication history. The prevalence of VRE by region was $26.1 \%$ (95\% CI: 10.7-50.9\%; $\left.I^{2}=41.65 \% ; P=0.113\right)$ in Addis Ababa, $15.0 \%$ (95\% CI: 6.9-29.6\%; $I^{2}=79.39 \% ; P<0.001$ ) in Amhara, 9.0\% (95\% CI: $2.8-25.7 \% ; I^{2}=71.49 \% ; P=0.015$ ) in Oromia and $1.9 \%$ (95\% CI: $0.1-23.1 \%)$ in Southern nations, nationalities and peoples region (SNNPR) (Table 3, Fig. 4). The prevalence of VRE pooled from studies conducted in the period before 2015 was $16.5 \%$ and that of the post-2015 was $16.3 \%$; which indicates unchanged trend of VRE infections in Ethiopia. On the other hand, the pooled prevalence of VRE from studies which used disc diffusion to determine AST was $16.9 \%$ and it was $7.9 \%$ when AST was measured by dilution/minimum inhibitory concentrations (MICs). Relatively; high rates of VRE were isolated from urine (37.3\%) and blood (22.0\%) specimens. Use of multisite specimens did not increase the isolation rate of enterococci. Unpublished studies reported high rate of VRE than published studies (31.9\% Vs. 11.3\%; respectively) (Table 3). 


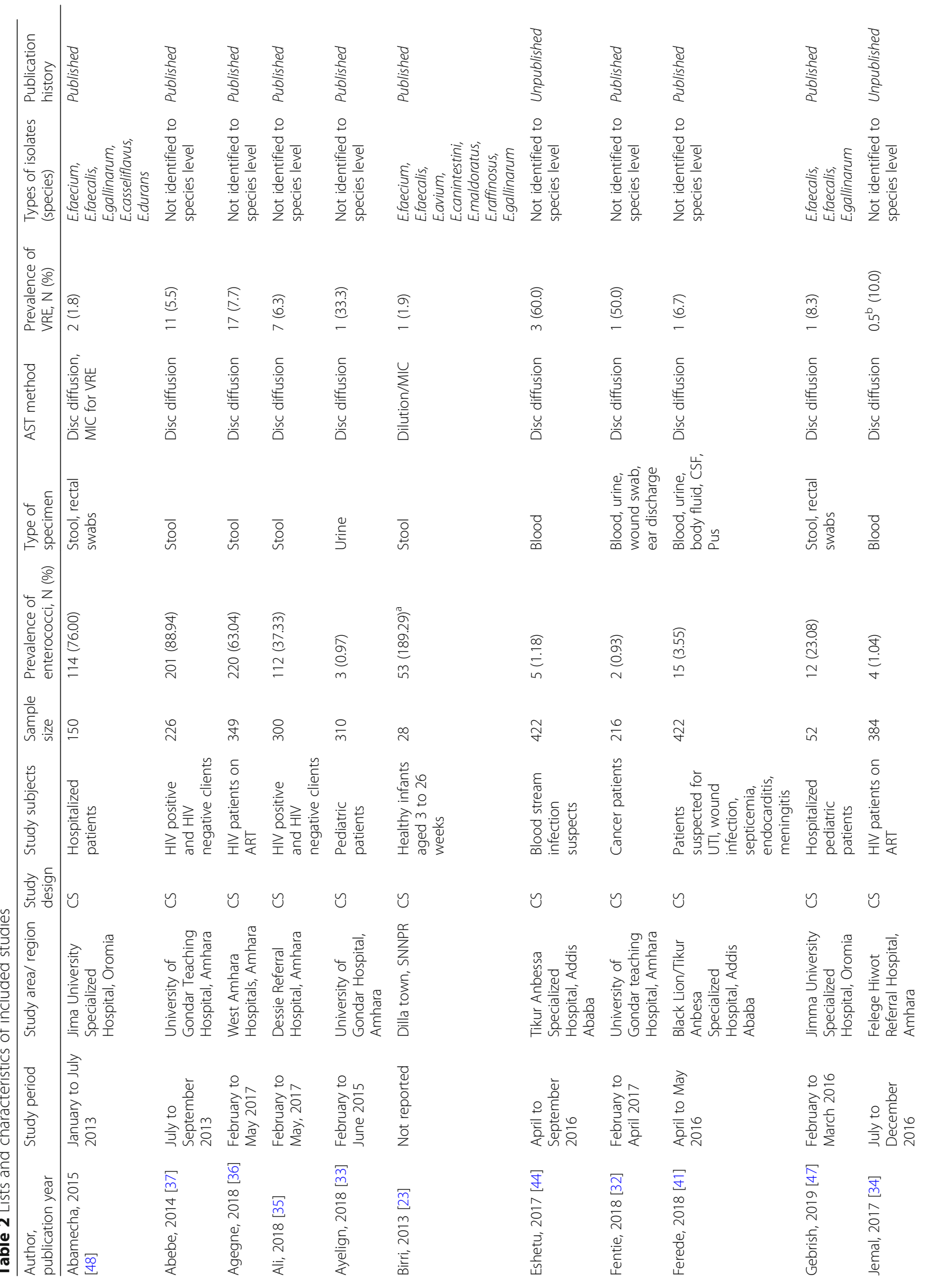




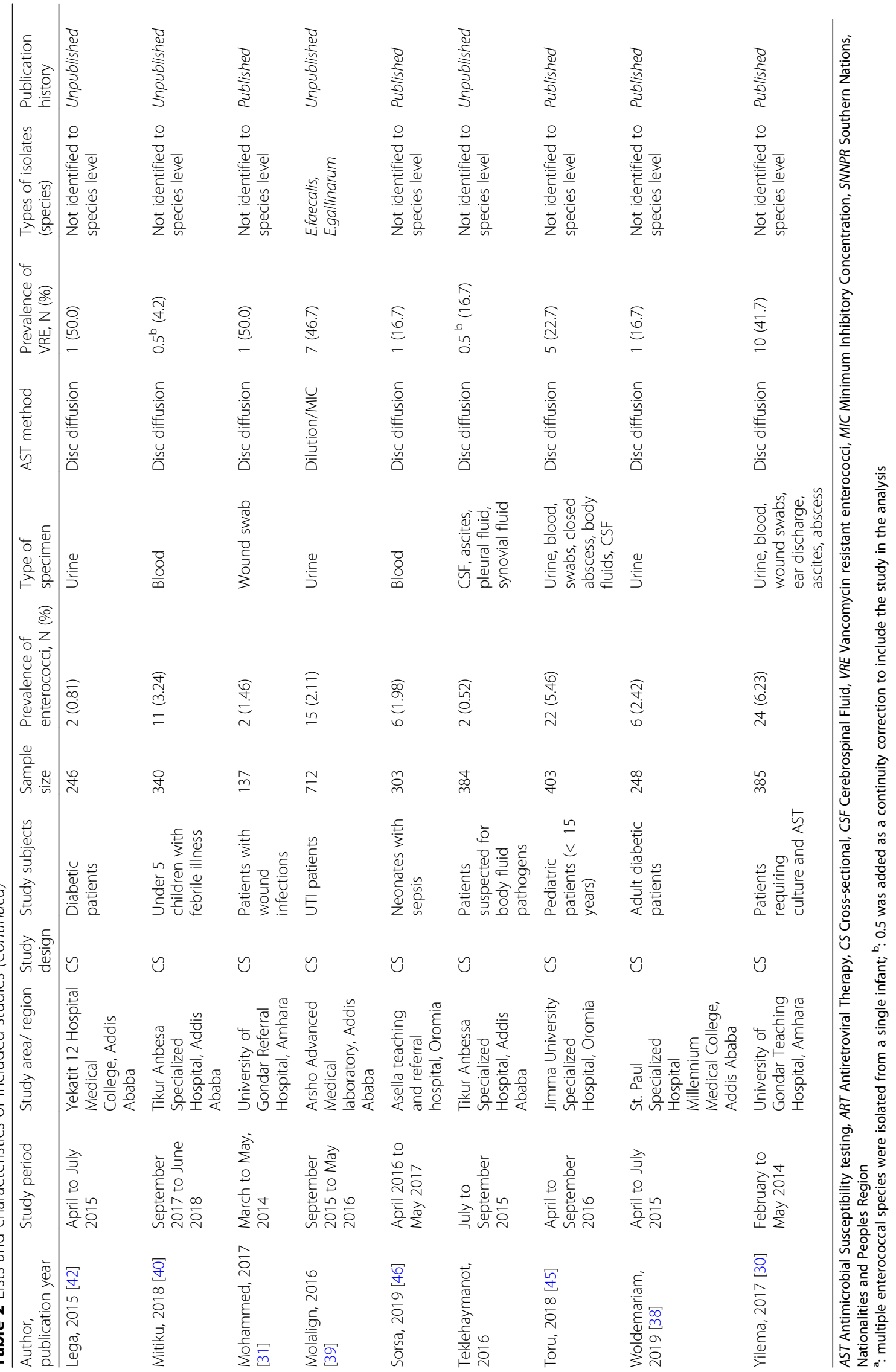




\section{Meta analysis}

\begin{tabular}{|c|c|c|c|c|}
\hline \multirow[t]{2}{*}{ Study name } & \multicolumn{4}{|c|}{ Statistics for each study } \\
\hline & $\begin{array}{c}\text { Event } \\
\text { rate }\end{array}$ & $\begin{array}{l}\text { Lower } \\
\text { limit }\end{array}$ & Upper & p-Value \\
\hline Abarnecha, 2015 & 0.018 & 0.004 & 0.067 & 0.000 \\
\hline Abebe, 2014 & 0.055 & 0.031 & 0.096 & 0.000 \\
\hline Agegne, 2018 & 0.077 & 0.049 & 0.121 & 0.000 \\
\hline Ali, 2018 & 0.083 & 0.030 & 0.125 & 0.000 \\
\hline Ayelign, 2018 & 0.333 & 0.043 & 0.846 & 0.571 \\
\hline Birri, 2013 & 0.019 & 0,003 & 0.122 & 0.000 \\
\hline Eshetu, 2017 & 0.600 & 0.200 & 0.900 & 0.657 \\
\hline Fentie, 2018 & 0.500 & 0.059 & 0.941 & 1.000 \\
\hline Ferede, 2018 & 0.067 & 0.009 & 0.352 & 0.011 \\
\hline Gebrish, 2019 & 0.083 & 0.012 & 0.413 & 0.022 \\
\hline Jemal, 2017 & 0.100 & 0006 & 0.674 & 0.140 \\
\hline Lega, 2015 & 0.500 & 0.059 & 0.941 & 1.000 \\
\hline Mitiku, 2018 & 0.042 & 0.003 & 0.425 & 0.030 \\
\hline Mohammed, 2017 & 0.500 & 0.059 & 0.941 & 1.000 \\
\hline Molalign, 2016 & 0.467 & 0.241 & 0.707 & 0.796 \\
\hline Sorsa, 2019 & 0.167 & 0.023 & 0.631 & 0.142 \\
\hline Teidehaymanot, 2016 & 0.167 & 0.010 & 0.806 & 0.299 \\
\hline Toru, 2018 & 0.227 & 0098 & 0.444 & 0.016 \\
\hline Woldemariam, 2019 & 0.167 & 0.023 & 0.631 & 0.142 \\
\hline Yilema, 2017 & 0.417 & 0.241 & 0.617 & 0.416 \\
\hline Overall & 0.148 & 0.087 & 0.243 & 0.000 \\
\hline
\end{tabular}

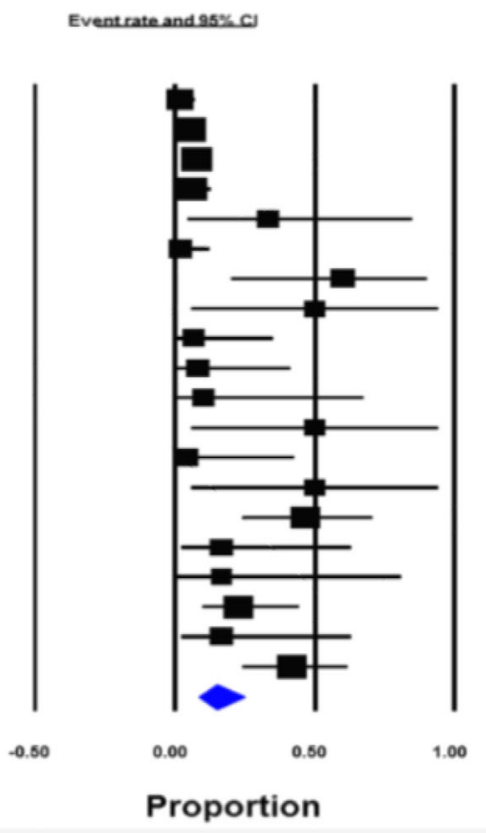

Fig. 2 Forest plot showing the pooled prevalence of VRE in Ethiopia

\section{Antimicrobial resistant enterococci}

The resistance profile of enterococci was also pooled for antimicrobials other than Vancomycin. Resistance rates were pooled if at least two studies reported on a specific bacterium-antibiotic combinations. High level of resistance was observed to all classes of tested antimicrobials except to Daptomycin and Linezolid. The pooled resistance rate of enterococci to Daptomycin was 3.2\% (95\% CI; $0.5-19.7 \%)$ and that of Linezolid was $9.9 \%$ (95\% CI; $2.8-29.0 \%)$. The pooled resistance rate to other antimicrobials was $60.7 \%$ (95\% CI; 39.2-78.3\%) to Penicillin, 56.5\% (95\% CI; 49.6-63.2\%) to Amoxicillin, 53.7\% (95\% a.

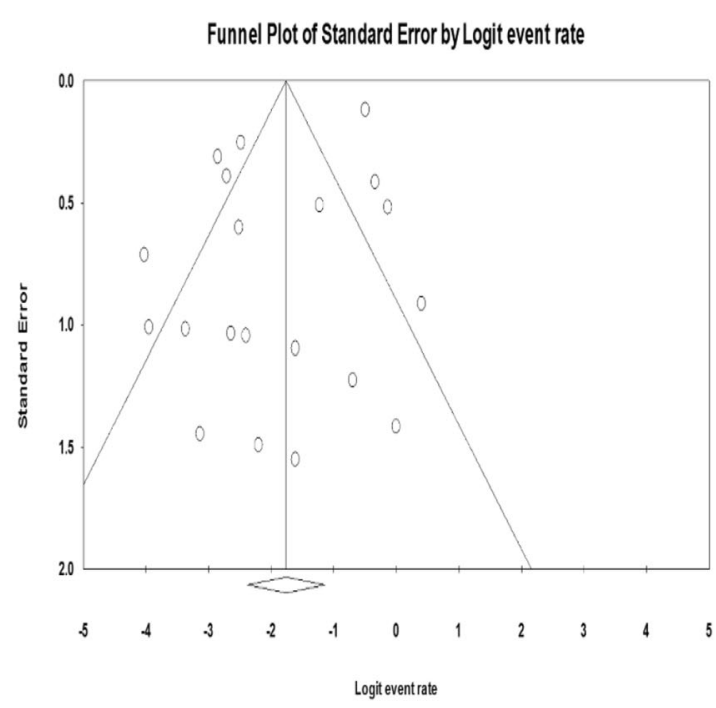

b.

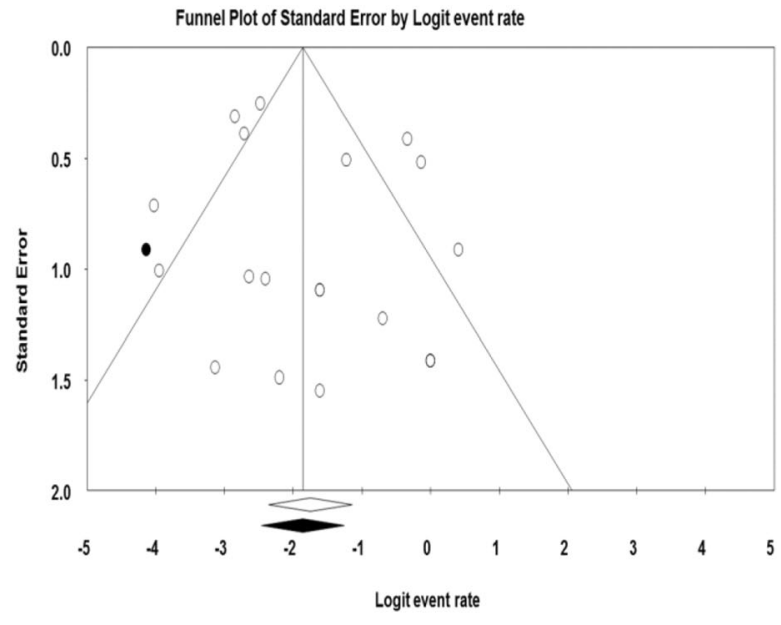

Fig. 3 Funnel plot showing publication bias; before (a) and after (b) the Trim-and-Fill method is applied 
Table 3 Pooled prevalence of VRE by subgroups

\begin{tabular}{|c|c|c|c|c|c|c|}
\hline Subgroups & Numbers of studies & No of enterococci isolates tested, $\mathrm{N}$ & Pooled prevalence of VRE, N (\%) & $95 \% \mathrm{Cl}$ & $P^{2}$ & $P$-value \\
\hline \multicolumn{7}{|l|}{ Region } \\
\hline Addis Ababa & 7 & 56 & $13(26.1)$ & $10.7-50.9$ & 41.65 & 0.113 \\
\hline Amhara & 8 & 568 & $38(15.0)$ & $6.9-29.6$ & 79.39 & $<0.001$ \\
\hline Oromia & 4 & 154 & $19(9.0)$ & $2.8-25.7$ & 71.49 & 0.015 \\
\hline SNNPR & 1 & 53 & $1(1.9)$ & $0.8-19.8$ & - & - \\
\hline \multicolumn{7}{|l|}{ Study period ${ }^{a}$} \\
\hline Before/in 2015 & 8 & 354 & $27(16.5)$ & $6.5-31.5$ & 81.09 & $<0.001$ \\
\hline After 2015 & 11 & 424 & $43(16.3)$ & $7.6-31.3$ & 69.20 & $<0.001$ \\
\hline \multicolumn{7}{|l|}{ AST method } \\
\hline Disc diffusion & 17 & 649 & $61(16.9)$ & $9.3-28.9$ & 66.89 & $<0.001$ \\
\hline Dilution/MIC & 3 & 182 & $10(7.9)$ & $1.9-27.6$ & 91.88 & $<0.001$ \\
\hline \multicolumn{7}{|l|}{ Type of specimen } \\
\hline Stool & 5 & 598 & $37(5.9)$ & $2.8-11.7$ & 0.00 & 0.629 \\
\hline Urine & 4 & 26 & $10(37.3)$ & $15.8-63.3$ & 0.00 & 0.665 \\
\hline Blood & 4 & 26 & $4(22.0)$ & $6.9-51.9$ & 45.06 & 0.141 \\
\hline Wound swab & 1 & 2 & $1(50.0)$ & - & - & - \\
\hline Multi-site $^{b}$ & 6 & 179 & $19(16.8)$ & $8.0-31.9$ & 77.87 & $<0.001$ \\
\hline \multicolumn{7}{|l|}{ Publication history } \\
\hline Published & 14 & 792 & $60(11.3)$ & $6.4-19.2$ & 72.86 & $<0.001$ \\
\hline Unpublished & 6 & 39 & $11(31.9)$ & $12.9-59.7$ & 25.82 & 0.241 \\
\hline
\end{tabular}

${ }^{a}$ One study did not report its study period; ${ }^{b}$ Studies used more than one type of specimen to isolate enterococci; MIC: Minimum Inhibitory Concentration

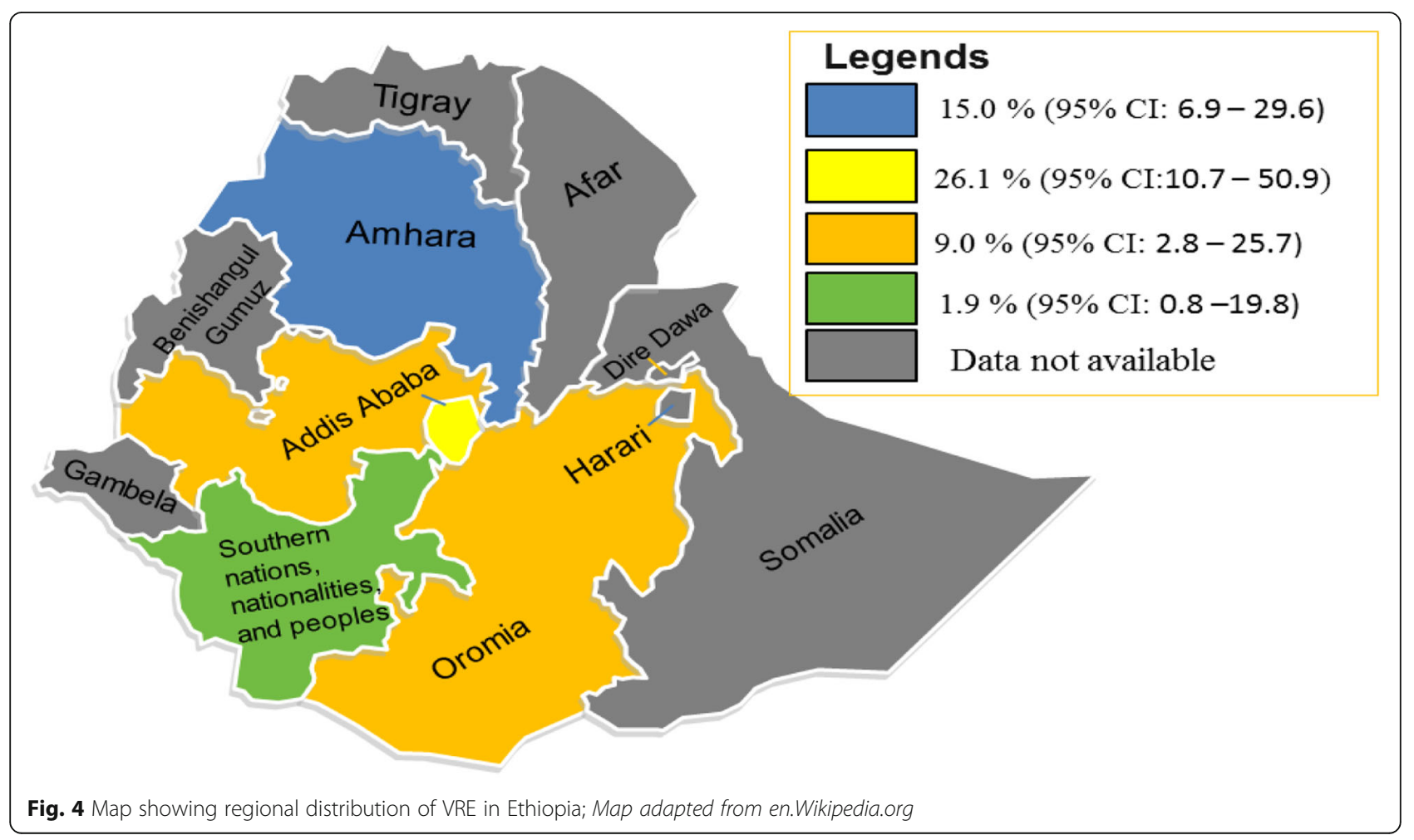


CI; $35.8-70.7 \%$ ) to Tetracycline, $55.1 \%$ (95\% CI; $22.2-$ $84.9 \%$ ) to Doxycycline, and $49.6 \%$ (95\% CI; 36.5-62.7\%) to Erythromycin. Studies reporting resistance to three or more antimicrobials were also pooled to estimate the prevalence of multidrug resistant (MDR) enterococci in Ethiopia. Hence; the overall prevalence of MDR enterococci was $63.0 \%\left(95 \% \mathrm{CI} ; 48.6-75.4 \% ; \mathrm{I}^{2}=90.27 \%\right.$; $P<0.001)$ (Table 4).

\section{Discussion}

Determining the prevalence of antibiotic resistance is an important step in the formulation of interventions to control emergence and transmission of resistant pathogens. In recent years, an increase in invasive VRE infections have been reported elsewhere in the worldwide [13, 17, 25, 49]. Although antimicrobial resistance surveillance centers were established and priority surveillance pathogens were identified to prevent the spread of drug resistance in Ethiopia, VRE were not included in the lists of priority pathogens. A previous systematic review [26] reporting the growing challenges of antibacterial resistance in Ethiopia had not assessed the burden of drug resistant enterococci. The prevalence of VRE has been reported by several studies in Ethiopia but a comprehensive review covering different parts of Ethiopia has not been conducted. This systematic review and meta-analysis was conducted to estimate the pooled prevalence of VRE and antimicrobial resistance profile of enterococci in Ethiopia.

Twenty studies reporting the prevalence and/or number of VRE isolates were included in this study. Majority (80\%) of the included studies failed to report the isolated enterococci at species level and simply highlighted the corresponding antimicrobial resistance profile. This might be due to poor laboratory capacity to identify species of enterococci. This indirectly indicates the potential existence of drug resistant enterococci in health care settings in Ethiopia and possible spread to the communities unless appropriately maintained., Although there was considerable methodological difference between studies, they were pooled for the purpose of this review. Therefore; the pooled prevalence of VRE in Ethiopia was estimated at $14.8 \%$. This estimate is comparable with reports from Iran (14, 18.75\%) [50, 51].

On the other hand, our finding was lower than studies reported from North America (21\%), Asia (24\%) and Europe (20\%) [52]. Another study from Iran reported high rate of VRE (48.9\%) among hospitalized patients [53]. These differences might be related with study population that hospitalized and critically ill patients are more likely to acquire VRE $[13,54]$ than the largely non-hospitalized study populations pooled in our analysis. In addition, the study period may contribute for the high rate of isolation in these countries. The studies were also conducted in the 1990's and 2000's following the first reports of VRE [21,

Table 4 Pooled resistance profile of enterococcal isolates in Ethiopia

\begin{tabular}{|c|c|c|c|c|c|c|}
\hline Antibiotics & No of studies & No of enterococci isolates tested, $\mathrm{N}$ & Pooled resistance N, (\%) & $95 \% \mathrm{Cl}$ & $P^{2}(\%)$ & $P$-value \\
\hline Amoxicillin & 2 & 203 & $115(56.5)$ & $49.6-63.2$ & 0.00 & 0.382 \\
\hline Amox-clavulanate & 2 & 225 & $71(45.3)$ & $13.9-80.9$ & 92.37 & $<0.001$ \\
\hline Ampicillin & 16 & 807 & $344(44.5)$ & $29.2-61.0$ & 90.83 & $<0.001$ \\
\hline Chloramphenicol & 12 & 777 & $188(32.9)$ & $20.8-47.8$ & 87.24 & $<0.001$ \\
\hline Ceftriaxone & 2 & 8 & $4(50.0)$ & $20.0-80.0$ & 0.00 & $>0.05$ \\
\hline Ciprofloxacin & 17 & 765 & $266(36.5)$ & $27.0-47.3$ & 75.30 & $<0.001$ \\
\hline Clindamycin & 4 & 224 & $59(26.9)$ & $21.5-33.2$ & 0.00 & 0.478 \\
\hline Daptomycin & 2 & 29 & $0.5(3.2)^{a}$ & $0.5-19.7$ & 0.00 & 0.974 \\
\hline Doxycycline & 3 & 254 & $85(55.1)$ & $22.2-84.0$ & 90.21 & $<0.001$ \\
\hline Erythromycin & 14 & 780 & $374(49.6)$ & $36.5-62.7$ & 86.19 & $<0.001$ \\
\hline Gentamycin & 10 & 533 & $248(37.7)$ & $22.2-56.1$ & 88.86 & $<0.001$ \\
\hline Linezolid & 2 & 30 & $2(9.9)$ & $2.8-29.0$ & 0.00 & 0.336 \\
\hline Nitrofurantoin & 9 & 404 & $117(31.5)$ & $23.4-41.0$ & 38.76 & 0.110 \\
\hline Norfloxacin & 5 & 350 & $100(39.9)$ & $18.6-66.6$ & 90.21 & $<0.001$ \\
\hline Penicillin & 8 & 343 & $181(60.7)$ & 39.9-78.3 & 86.63 & $<0.001$ \\
\hline Streptomycin & 3 & 179 & $74(36.8)$ & $10.4-73.1$ & 91.62 & $<0.001$ \\
\hline Tetracycline & 9 & 450 & $199(53.7)$ & $35.8-70.7$ & 86.95 & $<0.001$ \\
\hline SXT & 10 & 241 & $104(39.1)$ & $21.48-59.6$ & 45.58 & 0.088 \\
\hline MDR - enterococci & 20 & 825 & $543(60.0)$ & $42.9-75.0$ & 90.27 & $<0.001$ \\
\hline
\end{tabular}

SXT Trimethoprim-Sulfamethoxazole, MDR Multidrug resistance

${ }^{a}$ Continuity correction (0.5) is added to the study 
22]; while all of the studies included in our analysis were done in the 2010's where clinical use of Vancomycin was being discouraged [11].

In contrast, higher rates of VRE was observed in our study than reports from Singapore (9.3\%) [55], Germany (9.8\%) [49], Iran (9.4\%) [56] and United Kingdom (9.2\%) [57]. Different factors were identified as risk factors for acquiring VRE infections including previous hospitalization, patient transfer, urinary catheters, critical illnesses, underlying diseases, contact with VRE patients and inappropriate use of antibiotics $[54,55,58,59]$; all of which could contribute for the high prevalence of VRE in Ethiopia. Generally, infections and colonization with VRE were reported to be associated with health care contacts [18]. This could be true in settings where infection control knowledge, attitudes and practices among healthcare workers is poor in Ethiopia [60]. High frequency of inappropriate use of antibiotics and empirical therapies by healthcare professionals was also reported in Eastern Ethiopia [61]. In addition, the antimicrobial susceptibility testing method was based chiefly on disc diffusion and resistance was defined following the CLSI guideline.

Regional prevalence of VRE was also estimated. The highest estimated prevalence was obtained from Addis Ababa (26.1\%); almost two times higher than Amhara (15.0\%) and three times higher than Oromia (9.0\%). This regional difference might be attributed by different study settings (hospital set up), study period, study population, variation in antibiotic use, method of antimicrobial susceptibility testing and type of specimens used to isolate enterococci. Stool, urine and blood were the most common specimens from which VRE were isolated. This is not surprising because enterococci have been reported as the most common organisms isolated from intestinal tract, urinary tract and blood stream infections $[5,8,15,20,48,52,57,62]$.

Enterococci are not only resistant to Vancomycin but also to other commonly used antimicrobials including Penicillin, Amoxicillin, Doxycycline, Tetracycline, Erythromycin, Daptomycin, Linezolid and others (see Table 4 above). Multidrug resistant (MDR) enterococci were also observed that could pose a critical health problem in patients and health care settings in Ethiopia. As there is no specific recommendation for the antimicrobial prescription of VRE and a follow up surveillance is not conducted at different health care centers where the studies included in this review were conducted, the prevalence of VRE is expected to continuously increase. With these concerns in mind, there has been success stories in treating VRE infections with Daptomycin and Linezolid [62]. In our analysis however; resistance to Daptomycin and Linezolid was observed in about 3.2 and $9.9 \%$ of enterococcal isolates, respectively. Although it requires strong studies, our analysis indicated that these drugs may select vancomycin resistant strains in some potentially pathogenic enterococci through antibiotic selection pressure as they showed some sort of resistance to Daptomycin and Linezolid.

\section{Strengths and limitations of the study}

A comprehensive search with clear inclusion and exclusion criteria was used, examined commonly used specimens and methods of susceptibility testing, and included unpublished studies retrieved from Addis Ababa University repository. The Trim-and-Fill method was applied to asymmetric funnel plots to produce adjusted estimates. There were a number of limitations in the depth and breadth of data. First; inability to report pooled estimates of VRE at species level due to the paucity of included studies reporting enterococci at species level. Second; the definition of VRE was not consistent across studies and different AST methods were combined limiting comparability and strength of this analysis. Third; data was not available from $54.5 \%$ of the regions, outside health care setting and non-human studies were excluded that may be difficult to generalize the pooled results. Fourth; combing resistance results from different patients across different regions might pool out the peaks of resistance in some settings. Lastly; the study protocol was not registered at PROSPERO.

\section{Conclusion}

The prevalence of VRE and drug resistant enterococci are on the rise in Ethiopia. Enterococcal isolates showed resistance to one or more of the commonly prescribed drugs in different or the same drug lines. Multidrug resistant (MDR) enterococci were also found. Although the rates were low, the emergence of resistance to Daptomycin and Linezolid is an alarm for searching new ways for the treatment and control of VRE infections. This review provides data about the current burden of VRE in Ethiopia and showed gaps that would be addressed in future studies to maintain the spread of VRE infections. Adherence to antimicrobial stewardship, comprehensive testing and ongoing monitoring of VRE infections in the health care settings are required.

\section{Supplementary information}

Supplementary information accompanies this paper at https://doi.org/10. 1186/s12879-020-4833-2.

Additional file 1. The Joanna Briggs Institute (JBI) Critical Appraisal Checklist for Studies Reporting Prevalence Data

Additional file 2. Quality of the included 20 studies evaluated by JBI critical appraisal checklists

\section{Abbreviations}

AMR: Antimicrobial resistance; ART: Antiretroviral Therapy; AST: Antimicrobial Susceptibility Testing; CLSI: Clinical and Laboratory Standards Institute; EPHI: Ethiopian Public Health Institute; JBI: Joanna Biggs Institution; MDR: Multi Drug resistance; MIC: Minimum Inhibitory Concentration; SXT: Trimethoprim-Sulfamethoxazole; VRE: Vancomycin Resistant Enterococci; WHO: World Health Organization 


\section{Acknowledgements}

Not applicable.

\section{Authors' contributions}

AM: Conceived and designed the study; select and assess quality of studies; extracted and analyzed data; interpreted results; and drafted the manuscript. TA: select and assess quality of studies; extracted data and interpret results. CG: interpret results and review the manuscript. All authors read and approved the manuscript.

\section{Funding}

The authors declare that they did not receive any funding from any source.

\section{Availability of data and materials}

The datasets used and/or analyzed during the current study are included in the manuscript.

\section{Ethics approval and consent to participate}

Not applicable

\section{Consent for publication}

Not applicable.

\section{Competing interests}

The authors declare that they have no competing interests.

\section{Author details}

'Department of Medical Laboratory Science, College of Medicine and Health Sciences, Bahir Dar University, Bahir Dar, Ethiopia. ${ }^{2}$ Department of Medical Laboratory Science, College of Health Sciences, Debre Tabor University, Debre Tabor, Ethiopia.

Received: 26 September 2019 Accepted: 28 January 2020

Published online: 11 February 2020

\section{References}

1. Francesca Prestinaci, P.P., Annalisa Pantosti, Antimicrobial resistance: a global multifaceted phenomenon. Pathogens and Global Health. 2015;109(7):309-18.

2. Marshall, S.B.L.B., Antibacterial resistance worldwide: causes, challenges and responses. Nature Medicine Supplement. 2004;10(12):122-29.

3. Marianne Frieri KK, Boutin A. Antibiotic resistance. J Infection Public Health 2017;10:369-78.

4. Institute, E.P.H., Ethiopia antimicrobial resistance surveillance, annual report 2017/18. 2018

5. Banla LI, Salzman NH, Kristich CJ. Colonization of the mammalian intestinal tract by enterococci. Curr Opin Microbiol. 2019:47:26-31.

6. Yitbarek Getachew LH, Zakaria Z, Aziz SA. Genetic Variability of VancomycinResistant Enterococcus faecium and Enterococcus faecalis Isolates from Humans, Chickens, and Pigs in Malaysia. Appl Environ Microbiol. 2013;79(15):4528-33.

7. Bekele Hailu MA. Distribution of drug resistance among enterococci and Salmonella from poultry and cattle in Ethiopia. Trop Anim Health Prod. 2010;42:857-64.

8. Ana L. Flores-Mireles, J.N.W., Michael Caparon and Scott J. Hultgren, Urinary tract infections - epidemiology, mechanisms of infection and treatment options. Nature Reviews Microbiol. 2015;13:269-84.

9. Yomna A. Hashem, H.M.A., Tamer M. Essam, Aymen S. Yassin \& Ramy K. Aziz, Biofilm formation in enterococci - genotype-phenotype correlations and inhibition by vancomycin. Sci Rep. 2017;7(5733):1-12.

10. Wagner T, Joshi B, Janice J, Askarian F, Škalko-Basnet N, Hagestad OC, Mekhlif A, Wai SN, Hegstad K, Johannessen M. Enterococcus faecium produces membrane vesicles containing virulence factors and antimicrobial resistance related proteins. J Proteome. 2018;187:28-38.

11. G Werner, Coque TM, Hammerum AM, et al, Emergence and spread of vancomycin resistance among enterococci in Europe. Eurosurveillance. 2008;13(47):1-11.

12. Nade'ge Bourgeois-Nicolaos, C.M., Nicole Mangeney, Marie-Jos 'e Butel \& Florence Doucet-Populaire, Comparative study of vanA gene transfer from Enterococcus faecium to Enterococcus faecalis and to Enterococcus faecium in the intestineof mice. FEMS Microbiol Lett, 2005. 254(2006): p. 27-33.
13. Daniel J. Adams, Eberly MD, Goudie A, Cade M. Nylund, Rising VancomycinResistant Enterococcus Infections in Hospitalized Children in the United States. Hospital Pediatrics. 2016;6(7):404-11.

14. Arias CA, Murray BE., The rise of the Enterococcus - beyond vancomycin resistance. Nature Reviews Microbiol. 2012;10:266-78.

15. CA DG, Zimmer SM, Klein M, Jernigan JA. Comparison of Mortality Associated with Vancomycin-Resistant and Vancomycin-Susceptible Enterococcal Bloodstream Infections: A Meta-analysis. Clin Infect Dis. 2005:41:327-33.

16. K. Hegstad, Mikalsen T, Coque TM, G. Werner and A. Sundsfjord, Mobile genetic elements and their contribution to the emergence of Antimicrobial resistant Enterococcus faecalis and Enterococcus faecium. Clin Microbiol Infect, 2010. 16(6): p. 541-554.

17. Schroder UC, et al. Detection of vancomycin resistances in enterococci within 3 (1/2) hours. Sci Rep. 2015;5:8217.

18. Bernard C. Camins, M.M.F., John J. Jernigan, Susan M. Ray, James P. Steinberg Henry M. Blumberg, A population-based investigation of invasive vancomycinresistant enterococcus infection in metropolitan Atlanta, Georgia, and predictors of mortality. Infect Control Hosp Epidemiol. 2007;28(8):983-91.

19. Gearhart M. M.J., Rudich S, Thomas M, Wetzel D, Solomkin J, Hanaway MJ, Aranda-Michel J, weber F, Trumball L, bass M, Zavala E, Woodle ES, Buell JF, Consequences of vancomycin-resistant Enterococcus in liver transplant recipients: a matched control study. Clin Transpl. 2005;19:711-6.

20. A. L. Y. Cheah, T.S., D. Liew6, T. Peel, B. P. Howden, D. Spelman, M. L Grayson, R. L. Nation and D. C. M. Kong, Enterococcal bacteraemia: factors influencing mortality, length of stay and costs of hospitalization. Clinical Microbiology and Infection. 2913;19(4):E181-9.

21. Rice, L.B., Emergence of Vancomycin-Resistant Enterococci. Emerg Infect Dis. 2001:7(2):183-7.

22. Murray, B.E., Diversity among Multidrug-Resistant Enterococci. Emerg Infect Dis. 1998:4(1):37-47.

23. Birri DJ, et al. Bacteriocin production, antibiotic susceptibility and prevalence of haemolytic and gelatinase activity in faecal lactic acid bacteria isolated from healthy Ethiopian infants. Microb Ecol. 2013;65(2):504-16.

24. $\mathrm{WHO}, \mathrm{Global}$ priority list of antibiotic-resistant bacteria to guide research, discovery, and development of new antibiotics 2017

25. Cornelius Remschmidt, C.S., Michael Behnke, Petra Gastmeier, Christine Geffers and Tobias Siegfried Kramer, Continuous increase of vancomycin resistance in enterococci causing nosocomial infections in Germany - 10 years of surveillance. Antimicrob Resist Infect Control. 2018;7(54).

26. Moges $F$, et al. The growing challenges of antibacterial drug resistance in Ethiopia. J Glob Antimicrob Resist. 2014;2(3):148-54.

27. Moher D, L.A., Tetzlaff J, Altman DG „The PRISMA Group (2009). Preferred Reporting Items for Systematic Reviews and Meta-Analyses: The PRISMA Statement. PLoS Med, 2009. 6(6): p. e1000097.

28. Munn Z, M.S., Lisy K, Riitano D, Tufanaru C., Methodological guidance for systematic reviews of observational epidemiological studies reporting prevalence and incidence data. Int J Evid Based Healthc. 2015:13(3):1-7.

29. Andrea C Tricco, C.H.N., Vladimir Gilca, Andrea Anonychuk, Ba' Pham and Shirra Berliner, Canadian oncogenic human papillomavirus cervical infection prevalence: Systematic review and meta-analysis BMC Infectious Diseases. 2011;11(235).

30. Yilema A, et al. Isolation of enterococci, their antimicrobial susceptibility patterns and associated factors among patients attending at the University of Gondar Teaching Hospital. BMC Infect Dis. 2017;17(1):276.

31. Mohammed A, et al. Bacterial Isolates and Their Antimicrobial Susceptibility Patterns of Wound Infections among Inpatients and Outpatients Attending the University of Gondar Referral Hospital, Northwest Ethiopia. Int J Microbiol. 2017;2017:8953829.

32. Fentie $\mathrm{A}$, et al. Bacterial profile, antibiotic resistance pattern and associated factors among cancer patients at University of Gondar Hospital, Northwest Ethiopia. Infect Drug Resist. 2018;11:2169-78.

33. Ayelign B, et al. Bacterial isolates and their antimicrobial susceptibility patterns among pediatric patients with urinary tract infections. Turk J Urol. 2018:44(1):62-9.

34. Mohabaw Jemal, A.B., Yesuf Adem, Bacterial bloodstream infections and their antimicrobial susceptiblity pattern among HIV/AIDS patients at Felege Hiwot Referral Hospital, Bahir Dar, Amahara regional state, Northwest Ethiopia. 2017.

35. Ali S, et al. Vancomycin-resistant enterococci and its associated risk factors among HIV-positive and -negative clients attending Dessie referral hospital, Northeast Ethiopia. Int J Microbiol. 2018;2018:4753460. 
36. Agegne $M$, et al. Magnitude of Vancomycin-resistant enterococci (VRE) colonization among HIV-infected patients attending ART Clinic in West Amhara government hospitals. Int J Microbiol. 2018;2018:7510157.

37. Abebe $W$, et al. Prevalence of vancomycin resistant enterococci and associated risk factors among clients with and without HIV in Northwest Ethiopia: a cross-sectional study. BMC Public Health. 2014;14:185.

38. Woldemariam HK, et al. Common uropathogens and their antibiotic susceptibility pattern among diabetic patients. BMC Infect Dis. 2019;19(1):43.

39. Tamirat Molalign AB. Spectrum and antibiotic susceptibility profile of bacteriuria isolated from patients attending arsho advanced medical laboratory with urinary tract infections by using VITEK 2 compact system, Addis Ababa, Ethiopia; 2016.

40. Mequanint Mitiku KD. Multi-drug resistant bacterial Isolates among septicemia suspected under five children in Tikur Anbesa specialized hospital, Addis Ababa Ethiopia; 2018.

41. Ferede ZT, et al. Prevalence and antimicrobial susceptibility pattern of Enterococcus species isolated from different clinical samples at black lion specialized teaching hospital, Addis Ababa, Ethiopia. BMC Res Notes. 2018; 11(1):793.

42. Tebarek Lega IA, Desta K, Kebede T. Bacterial Uropathogens and their Drug Resistance Pattern in Diabetic Patients Attending Yekatit 12 Hospita Medical College, Addis Ababa, Ethiopia; 2015.

43. Frehiwot Teklehaymanot KD, Hailu M. Bacterial profiles and their antimicrobial susceptibility patterns from body fluids at Tikur Anbessa Specialized Hospital, Addis Ababa, Ethiopia; 2016.

44. Seneshat Eshetu AB, Getachew T, Gizaw S. Bacterial profile and antimicrobial susceptibility pattern of blood culture isolates at Tikur Anbessa Specialized Hospital, Addis Ababa, Ethiopia; 2017.

45. Toru M, et al. Prevalence and phenotypic characterization of Enterococcus species isolated from clinical samples of pediatric patients in Jimma University specialized hospital, south West Ethiopia. BMC Res Notes. 2018;11(1):281.

46. Sorsa A, et al. Blood culture result profile and antimicrobial resistance pattern: a report from neonatal intensive care unit (NICU), Asella teaching and referral hospital, Asella, south East Ethiopia. Antimicrob Resist Infect Control. 2019;8:42.

47. Gebrish S., F.B., Asfaw T., Magnitude of Drug-resistant Enterococcus species from Intestinal Tracts of Hospitalized Pediatric Patients in Ethiopia. 2019.

48. Abamecha A, Wondafrash B, Abdissa A. Antimicrobial resistance profile of Enterococcus species isolated from intestinal tracts of hospitalized patients in Jimma, Ethiopia. BMC Res Notes. 2015:8:213.

49. Tobias Siegfried Kramer, C.R., Sven Werner, Michael Behnke, Frank Schwab, Guido Werner, Petra Gastmeier and Rasmus Leistner, The importance of adjusting for enterococcus species when assessing the burden of vancomycin resistance: a cohort study including over 1000 cases of enterococcal bloodstream infections. Antimicrob Resist Infect Control. 2018;7(133).

50. Ali Jahansepas, M.A.R., Alka Hasani, Yaeghob Sharifi, Marjan Rahnamaye Farzami, Alireza Dolatyar, and Mohammad Aghazadeh, Molecular Epidemiology of Vancomycin-Resistant Enterococcus faecalis and Enterococcus faecium Isolated from Clinical Specimens in the Northwest of Iran. Microb Drug Resist, 2018. 00(00).

51. Abbas Moghimbeigi MM, Dousti M, Kiani F, Sayehmiri F, Sadeghifard N, Nazari A. Prevalence of vancomycin resistance among isolates of enterococci in Iran: a systematic review and meta-analysis. Adolesc Health Med Ther. 2018;9:177-88.

52. Michail Alevizakos, A.G., Dimitrios Nasioudis, Katerina Tori, Myrto Eleni Flokas, and Eleftherios Mylonakis, Colonization With Vancomycin-Resistant Enterococci and Risk for Bloodstream Infection Among Patients With Malignancy: A Systematic Review and Meta-Analysis open forum infectious diseases, 2016.

53. Leili Shokoohizadeh, A.M.M., Mohammad Reza Zali, Reza Ranjbar, Masoud Alebouyeh, Türkan Sakinc and Liaqat Ali, High frequency distribution of heterogeneous vancomycin resistant Enterococcous faecium (VREfm) in Iranian hospitals. Diagn Pathol. 2013;8(163).

54. Mazuski, J.E., Vancomycin-Resistant Enterococcus: Risk Factors, Surveillance, Infections, and Treatment. Surg Infect. 2008;9(6):567-71.

55. Kok-Soong Yang Y-TF, Lee H-Y, Kurup A, Koh T-H, Koh D, Lim M-K. Predictors of Vancomycin-resistant Enterococcus (VRE) Carriage in the First Major VRE Outbreak in Singapore. Ann Acad Med Singap. 2007;36:379-83.

56. Emaneini M, Hosseinkhani F, Jabalameli F, Nasiri MJ, Dadashi M, Pouriran R, Beigverdi R. Prevalence of vancomycin-resistant Enterococcus in Iran - a systematic review and meta-analysis. Eur J ClinMicrobiol Infect Dis. 2016;35: 1387-92.
57. Liam Toner NP, Aliyu SH, Dev H, Lawrentschuk N, Al-Hayek S. Vancomycin resistant enterococci in urine cultures: Antibiotic susceptibility trends over a decade at a tertiary hospital in the United Kingdom. ICUrology. 2016;57: 129-34.

58. KUO-MING YEH, Siu LK, Chang JC, Chang FY., Vancomycin-Resistant Enterococcus (VRE) Carriage and Infection in Intensive Care Units. Microb Drug Resist. 2014;10(2):177-83.

59. Melissa Barger, E.B., Sol Pena, Wendy Mack and Tse-Ling Fong, VRE in cirrhotic patients. BMC Infect Dis. 2019;19(711).

60. Tenna A, Stenehjem EA, Margoles L, Kacha E, Blumberg HM, Kempker RR. Infection Control Knowledge, Attitudes, and Practices among Healthcare Workers in Addis Ababa, Ethiopia. Infect Control Hosp Epidemiol. 2013; 24(12):1289-96.

61. Tafa B, Endale A, Bekele D. Paramedical staffs knowledge and attitudes towards antimicrobial resistance in Dire Dawa, Ethiopia: a cross sectional study. Ann Clin Microbiol Antimicrob. 2017;16(1):64.

62. Jennifer D. Twilla, Finch CK, Justin B. Usery, Michael S. Gelfand, Joanna Q. Hudson, and Joyce E. Broyles, Vancomycin-Resistant Enterococcus Bacteremia: An evaluation of Treatment with Linezolid or Daptomycin. J Hosp Med. 2012;7(3):243-8.

\section{Publisher's Note}

Springer Nature remains neutral with regard to jurisdictional claims in published maps and institutional affiliations.
Ready to submit your research? Choose BMC and benefit from:

- fast, convenient online submission

- thorough peer review by experienced researchers in your field

- rapid publication on acceptance

- support for research data, including large and complex data types

- gold Open Access which fosters wider collaboration and increased citations

- maximum visibility for your research: over $100 \mathrm{M}$ website views per year

At BMC, research is always in progress.

Learn more biomedcentral.com/submissions 